\title{
Combined Effects of Hall Current and Heat Transfer on Peristaltic Transport of a Nanofluid in a Vertical Tapered Channel Through a Porous Medium
}

\author{
Ahmed I. Abdellateef and Syed Z. UI Haque*
}

\begin{abstract}
Department of Mathematics and Statistics, Caledonian College of Engineering, Sultanate of Oman, Muscat. University of Glasgow, Scotland, UK. *Email: hqzahoor@gmail.com.
\end{abstract}

\begin{abstract}
The influences of Hall currents and heat transfer on peristaltic transport of a nanofluid in a vertical porous tapered channel through a porous medium are investigated theoretically and graphically under the assumptions of low Reynolds number and long wavelength and the flow investigated is in a wavy frame of reference. Analytical solutions are obtained for temperature, axial velocity, stream function and pressure gradient. Graphical results are sketched for various embedded parameters.
\end{abstract}

Keywords: Peristaltic flow; Heat transfer; Nanofluid; Tapered channel; Hall currents and Porous medium.
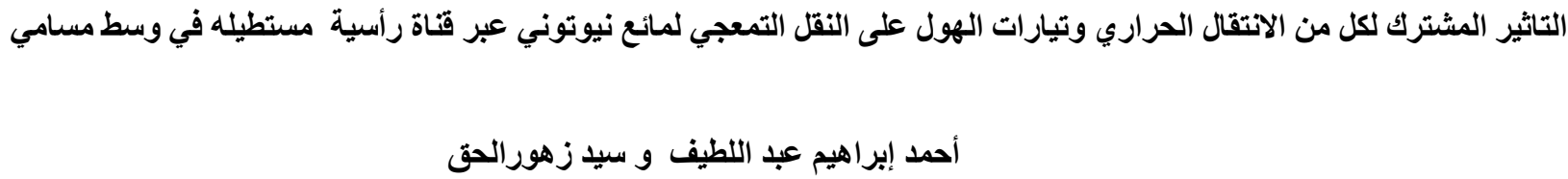

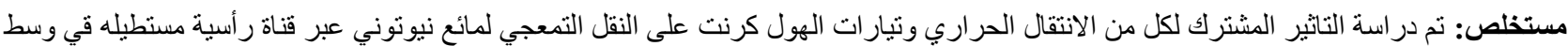

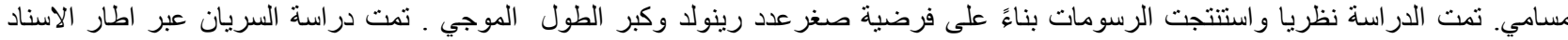

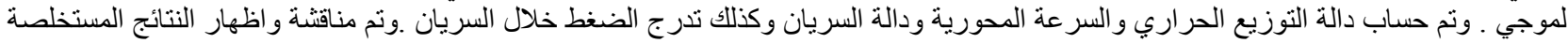

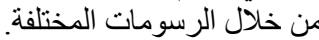

كلمات مفتاحية: نقل تمعجي ، انتقال حر اري ، مائع نانوي ،قناة متباعدة ، تبار ات هول و وسط مسامي.

\section{Introduction}

$\mathbf{M}$ ass transfer and heat transfer from different geometries embedded in a porous medium have many geophysical and engineering applications such as thermal insulations, drying of porous solids, cooling of nuclear reactors, underground energy transport, and crude oil extraction. The study of fluid flow through a porous medium and heat transfer constitute a fundamental study of nature and have great practical importance in several physical problems; these include porous heat exchangers, sewage of water in river beds, filtration and purification processes, for example. Their industrial importance has led to many experimental and analytical studies of flow and heat transfer in a porous medium in the presence of a magnetic field. McWhirter et al. [1], and Geindreau and Auriault [2] have discussed magnetohydrodynamic (MHD) flow through a porous medium in great details. The tapered channel is specially designed to assist in the management of subglottic, tracheal, and glottic stenosis. The superior end conforms to the contours of the glottis, while the larger inferior portion simultaneously acts as a stent for the trachea. The design of the tapered end is such that it projects up beyond the true vocal cords to the level of the laryngeal ventricle. Peristaltic flow is a phenomenon found in several industrial and physiological processes. Within the body peristaltic flows occur widely in fluid processes such as in urine transport from kidney to bladder through the ureter, transport of lymph in the lymphatic vessels, swallowed food through the esophagus, spermatozoa transport in the human reproductive tract, vaso motion of small blood vessels, etc. In addition to that, peristaltic pumping occurs in many practical applications involving biomechanical systems. The mechanism of peristaltic flow can be best explained by comparing the experimental clinical data and mathematical results. A perfect mathematical study can be served to many peristaltic flows occuring in the human body. Many research attempts had been made to understand peristaltic action in different situations. The first attempt was made by Latham [3] and some of the interesting investigations in the same direction are given in references [4-7]. Moreover, the flow through a porous medium has also attracted the attention of many 
researchers in the past few decades because of its important practical applications, as it occurs in sandstone, limestone, filtration of fluids and seepage of water in river beds, wood, and in human lungs, the bile duct, the gall bladder with stones, and in small blood vessels etc. Shehawey et al. [8] formulated a mathematical model for the peristaltic transport of a viscous incompressible fluid through a porous medium bounded by two porous plates and El Shehawey et al. [9] studied the peristaltic transport in an asymmetric channel through a porous medium. Vajravelu et al. [10] examined peristaltic flow through vertical porous tube. Srinvas and Kothandapani [11] investigated the influence of heat and mass transfer on MHD peristaltic flow through a porous space with compliant walls. Srinvas and Gayathri [12] continued this work and discussed the peristaltic transport of Newtonian fluid in an asymmetric channel of a porous medium with heat transfer. The Hall effect is important when the Hall parameter which is the ratio between the electron-cyclotron frequency and the electron-atom-collision frequency is high. This can occur if the collision frequency is low or when the magnetic field is high. This is a current trend in MHD because of the important influence of the electromagnetic force. There has been considerable study of Hall effects and heat transfer effects on flow to determine the efficiency of certain devices such as heat exchangers and power generators. Attia [13] examined unsteady Hartmann flow with heat transfer of a viscoelastic fluid taking the Hall effect into consideration. Asghar et al. [14] studied the effects of Hall current and heat transfer on flow due to the pull of an eccentric rotating disk. Hayat et al. [15] studied the Hall effects on peristaltic flow of a Maxwell fluid in a porous medium. Abo-Eldahab et al. [16, 17] investigated the effects of Hall and ion-slip currents on MHD peristaltic transport and couple stress fluid. For further information the references [18-25] provide additional source.

The aim of this paper is to investigate the effects of Hall current and heat transfer on peristaltic transport of a Newtonian nanofluid in a tapered channel through a porous medium.

\section{Nomenclature}

$\begin{array}{ll}a_{1}, b_{1} & \text { Wave amplitudes } \\ \mathrm{d}_{1}+\mathrm{d}_{2} & \text { Two-dimensional channel width } \\ \lambda & \text { Wavelength of the peristaltic wave } \\ c_{1} & \text { Wave speed } \\ \phi & \text { Phase difference } \\ \rho_{f} & \text { Density of the fluid } \\ \rho_{p} & \text { Density of the particle } \\ \bar{q} & \text { Velocity vector } \\ T_{s} & \text { Fluid temperature in static condition } \\ D / D t & \text { Material derivative } \\ \bar{p} & \text { Pressure } \\ \bar{C} & \text { Nanoparticle volume fraction } \\ D_{B} & \text { Brownian diffusion coefficient } \\ D_{T} & \text { Thermophoretic diffusion coefficient } \\ d & \text { Amplitude ratio }\end{array}$

$R_{e} \quad$ Reynolds number

$\delta \quad$ Dimensionless wave number

$m_{1} \quad$ Hall current parameter

M Magnetic field parameter

$\hat{k} \quad$ Thermal conductivity

$N_{t} \quad$ Thermophoresis parameter

$N_{b} \quad$ Brownian motion parameter

$G_{r} \quad$ Thermal Grashof number

$B_{r} \quad$ Local nanoparticle Grashof number

$\Delta p \quad$ Pressure rise

$F_{\lambda} \quad$ Friction force

$K \quad$ Porosity parameter

$\theta \quad$ Temperature distribution

$\sigma \quad$ Dimensionless nanoparticle volume fraction

\section{Formulation of the problem}

Consider the peristaltic transport of a nanofluid in a two-dimensional channel of width $\left(d_{1}+d_{2}\right)$. The flow is induced by propagating a sinusoidal wave with constant speed 'c' along the channel walls (as shown in Fig. 1). We denote the channel walls by $H_{1}$ and $H_{2}$.

$$
\begin{array}{ll}
H_{1}(\bar{X}, \bar{t})=d_{1}+K_{1} x+a_{1} \cos \left[\frac{2 \pi}{\lambda}\left(X-c_{1} t\right)\right] & \text { Right wall } \\
H_{2}(\bar{X}, \bar{t})=-d_{2}-K_{2} x-b_{1} \cos \left[\frac{2 \pi}{\lambda}\left(X-c_{1} t\right)+\varphi\right] & \text { Left wall }
\end{array}
$$

where $a_{1}$ and $a_{2}$ are the wave's amplitudes, $\lambda$ is the wave length, $c_{1}$ is the wave speed, and $\phi(0 \leq \phi \leq \pi)$ is the phase difference. It should be noted that $\phi=0$ corresponds to a symmetric channel with waves out of phase and that 


\section{COMBINED EFFECTS OF HALL CURRENT AND HEAT TRANSFER}

for $\phi=\pi$ the waves are in phase. Further, $d_{1}, d_{2}, a_{1}, b_{1}$ and $\phi$ satisfy the following inequality $a_{1}^{2}+b_{1}^{2}+2 a_{1} b_{1} \cos \phi \leq\left(d_{1}+d_{2}\right)^{2}$. The walls $Y=H_{1}$ and $Y=H_{2}$, are maintained at temperatures $T_{1}$ and $T_{2}$ respectively.

The governing equations for the flow and heat transfer of an incompressible nanofluid in the absence of body force are:

$$
\begin{gathered}
\bar{\nabla} \cdot \bar{q}=0, \\
\rho_{f} \frac{D \bar{q}}{D t}=-\bar{\nabla} \bar{p}+\mu \bar{\nabla}^{2} \bar{q}+\bar{f}, \\
(\rho c)_{f} \frac{D \bar{T}}{D t}=k \bar{\nabla}^{2} \bar{T}+(\rho c)_{p}\left[D_{B}(\bar{\nabla} C) \cdot(\bar{\nabla} \bar{T})+\frac{D_{T}}{\left(T_{0}\right)}(\bar{\nabla} \bar{T}) \cdot(\bar{\nabla} \bar{T})\right], \\
\frac{D \bar{C}}{D t}=D_{B} \bar{\nabla}^{2} \bar{C}+\frac{D_{T}}{\left(T_{0}\right)} \bar{\nabla}^{2} \bar{T},
\end{gathered}
$$

where $\rho_{f}$ is the density of the fluid, $\rho_{p}$ is the density of the particle, $\bar{q}$ is the velocity vector, $\bar{f}$ is the body force, $T_{s}$ is the fluid temperature in static condition, $\frac{D}{D t}$ represents the material derivative, $\bar{p}$ is the pressure , $\bar{C}$ is the nanoparticle volume fraction, $D_{B}$ is the Brownian diffusion coefficient and $D_{T}$ is the thermophoretic diffusion coefficient. We now introduce a wavy frame $(x, y)$ moving with velocity $c_{1}$ away from the fixed frame $(X, Y)$ by the transformation

$$
x=X-c_{1} t, y=Y, u=U-c_{1}, v=V, p(x)=P(X, t)
$$

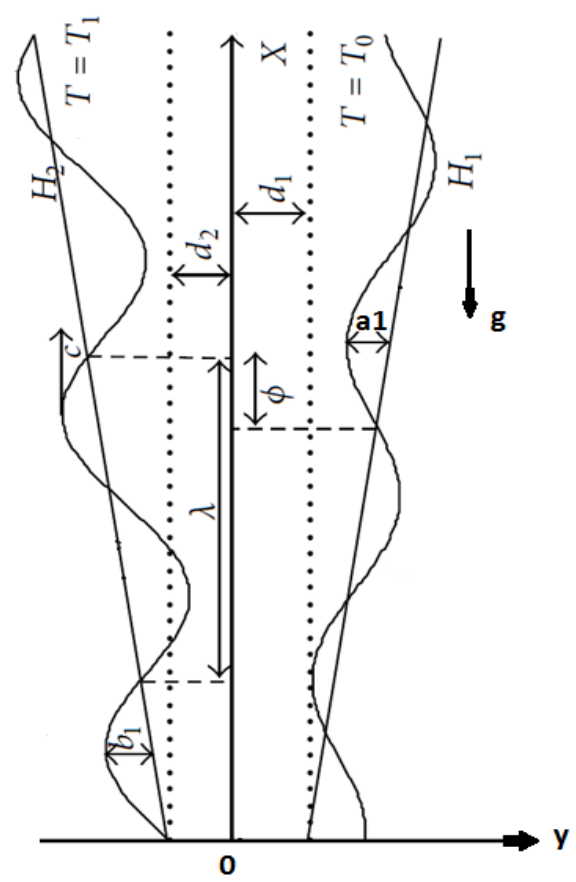

Figure 1. The simplified model geometry of the problem.

where $(u, v)$ are the velocity components in the wavy frame $(x, y), p$ and $P$ are pressures in wavy and fixed frame respectively. The pressure $p$ remains constant across any axial station of the channel under the assumption that the wavelength is large and the curvature effects are negligible. After using these transformations, the governing equations in steady case become:

$$
\frac{\partial \bar{u}}{\partial \bar{x}}+\frac{\partial \bar{v}}{\partial \bar{y}}=0
$$




$$
\begin{gathered}
\rho\left[\bar{u} \frac{\partial \bar{v}}{\partial \bar{x}}+\bar{v} \frac{\partial \bar{v}}{\partial \bar{y}}\right]=-\frac{\partial \bar{p}}{\partial \bar{y}}+\mu\left(\frac{\partial^{2} \bar{v}}{\partial \bar{x}^{2}}+\frac{\partial^{2} \bar{v}}{\partial \bar{y}^{2}}\right)-\frac{\mu}{\bar{k}_{1}} \bar{v}-\frac{\sigma B_{o}^{2}}{\left(1+m_{1}^{2}\right)}\left(m_{1} \bar{u}+\bar{v}\right) \\
\rho\left[\bar{u} \frac{\partial \bar{u}}{\partial \bar{x}}+\bar{v} \frac{\partial \bar{u}}{\partial \bar{y}}\right]=-\frac{\partial \bar{p}}{\partial \bar{x}}+\mu\left(\frac{\partial^{2} \bar{u}}{\partial \bar{x}^{2}}+\frac{\partial^{2} \bar{u}}{\partial \bar{y}^{2}}\right) \\
+\rho g \beta\left(T-T_{0}\right)+\rho g \beta^{*}\left(C-C_{0}\right)-\frac{\mu}{\bar{k}_{1}} \bar{u}+\frac{\sigma B_{o}^{2}}{\left(1+m_{1}^{2}\right)}\left(m_{1} \bar{v}-\bar{u}\right) \\
{\left[\bar{u} \frac{\partial \bar{T}}{\partial \bar{x}}+\bar{v} \frac{\partial \bar{T}}{\partial \bar{y}}\right]=\alpha\left(\frac{\partial^{2} \bar{T}}{\partial \bar{x}^{2}}+\frac{\partial^{2} \bar{T}}{\partial \bar{y}^{2}}\right)+\tau_{r}\left[D_{B}\left\{\left(\frac{\partial \bar{C}}{\partial \bar{y}} \frac{\partial \bar{T}}{\partial \bar{y}}\right)+\left(\frac{\partial \bar{C}}{\partial \bar{x}} \frac{\partial \bar{T}}{\partial \bar{x}}\right)\right\}+\right.} \\
{\left[\bar{u} \frac{\partial \bar{C}}{\partial \bar{x}}+\bar{v} \frac{\partial \bar{C}}{\partial \bar{y}}\right]=D_{B}\left(\frac{\partial^{2} \bar{C}}{\partial \bar{x}^{2}}+\frac{\partial \partial^{2} \bar{C}}{\partial \bar{y}^{2}}\right)+\frac{D_{T}}{\left(T_{0}\right)}\left(\frac{\partial^{2} \bar{T}}{\partial \bar{x}^{2}}+\frac{\partial^{2} \bar{T}}{\partial \bar{y}^{2}}\right)}
\end{gathered}
$$

where $\tau_{r}=\frac{(\rho c)_{p}}{(\rho c)_{f}}$ is the ratio between the effective heat capacity of the nanoparticle material and heat capacity of the fluid.

The corresponding no-slip boundary conditions are:

$$
\begin{aligned}
& \bar{u}=-c_{1}, \bar{C}=C_{1}, \quad \bar{T}=T_{1} \text { at } \bar{y}=\bar{H}_{1}, \\
& \bar{u}=-c_{1}, \bar{C}=C_{2}, \quad \bar{T}=T_{2} \text { at } \bar{y}=\bar{H}_{2} .
\end{aligned}
$$

Consider the following non-dimensional variables and parameters:

$$
\begin{gathered}
u=\frac{\bar{u}}{c_{1}}, v=\frac{\bar{v}}{\delta c_{1}}, x=\frac{\bar{x}}{\lambda}, y=\frac{\bar{y}}{d_{1}}, \delta=\frac{d_{1}}{\lambda}, p=\frac{d_{1}^{2} \bar{p}}{\mu \lambda c_{1}}, t=\frac{c \bar{t}}{\lambda}, h_{1}=\frac{\bar{H}_{1}}{d_{1}}, h_{2}=\frac{\bar{H}_{2}}{d_{1}}, \\
d=\frac{d_{2}}{d_{1}}, b=\frac{a_{2}}{d_{1}}, a=\frac{a_{1}}{d_{1}}, P_{r}=\frac{v}{\alpha}, R_{e}=\frac{c_{1} d_{1}}{v}, \theta=\frac{T-T_{s}}{T_{1}-T_{s}}, N_{t}=\frac{(\rho c)_{p} D_{T}\left(T_{1}-T_{s}\right)}{(\rho c)_{f} \alpha}, \\
\sigma=\frac{\left(\bar{C}-\overline{C_{0}}\right)}{\overline{C_{0}}}, N_{b}=\frac{(\rho c)_{p} D_{B} \overline{C_{0}}}{(\rho c)_{f} \alpha}, \alpha=\frac{\hat{k}}{(\rho c)_{f}}, G_{r}=\frac{g \beta d_{1}^{2}\left(T_{1}-T_{s}\right)}{v c}, \\
B_{r}=\frac{g \beta^{*} d_{1}^{2} \overline{C_{0}}}{v c}, k=\frac{\bar{k}_{1}}{a^{2}}, M=\frac{\sigma B_{0}^{2} d_{1}^{2}}{\mu}, k_{1}=\frac{K_{1}}{d_{1}}, k_{2}=\frac{K_{2}}{d_{1}}
\end{gathered}
$$

where $T_{s}$ - the fluid temperature in static condition, $d$ - the amplitude ratio, $R_{e}$ - the Reynolds number, $\delta$ - the dimensionless wave number, $m_{1}$ - the Hall current parameter, $M$ - the magnetic field parameter, $\hat{k}$-the thermal conductivity, and $N_{b}, N_{t}, B_{r}$ and $G_{r}$ are the Brownian motion parameter, thermophoresis parameter, local nanoparticle Grashof number, and local thermal Grashof number respectively.

With the help of Eq. (2.14) in Eqs.( 2.8)-(2.13) under the assumptions of long wavelength and low Reynold number approximation takes the form

$$
\begin{gathered}
\frac{\partial u}{\partial x}+\frac{\partial v}{\partial y}=0 \\
\frac{\partial p}{\partial y}=0 \\
\frac{\partial p}{\partial x}=\frac{\partial^{2} u}{\partial y^{2}}+G_{r} \theta+B_{r} \sigma-\left(\frac{1}{\bar{k}_{1}}+\frac{M}{\left(1+m_{1}^{2}\right)}\right) u \\
0=\frac{\partial^{2} \theta}{\partial y^{2}}+N_{b}\left(\frac{\partial \sigma}{\partial y} \frac{\partial \theta}{\partial y}\right)+N_{t}\left(\frac{\partial \theta}{\partial y}\right)^{2}
\end{gathered}
$$




$$
0=\frac{\partial^{2} \sigma}{\partial y^{2}}+\frac{N_{t}}{N_{b}} \frac{\partial^{2} \theta}{\partial y^{2}}
$$

The corresponding boundary conditions become:

$$
\begin{aligned}
& u=-1, \quad \theta=1, \quad \sigma=0, \quad y=h_{1}, \\
& u=-1, \quad \theta=m_{0}, \quad \sigma=0, y=h_{2},
\end{aligned}
$$

where $m_{0}=\left(T_{2}-T_{s}\right) /\left(T_{1}-T_{s}\right)$, the wall temperature ratio.

The solution of Eqs. $(2.18,2.19)$, valid in $h_{2} \leq y \leq h_{1}$ satisfying the corresponding boundary conditions (20) is given by

$$
\begin{aligned}
& \theta=1+A_{1}\left(e^{-s_{1} N_{b} y}-e^{-s_{1} h_{1} N_{b}}\right) \\
& \sigma=\frac{N_{t}}{N_{b}}(1-\theta)+s_{1}\left(y-h_{1}\right)
\end{aligned}
$$

By substituting Eqs. (2.21)-(2.22) in Eq. (2.17) and solving it analytically as a second order ordinary differential equation, we get a solution in the form,

\section{Rate of volume flow}

$$
u(x)=c_{2}+c_{3} y+c_{4} e^{-\sqrt{A_{2} y}}+c_{5} e^{\sqrt{A_{2} y}}+c_{6} e^{-s_{1} N_{b} y}
$$

The instantaneous volume flow rate in the fixed frame is given by

$$
Q=\int_{\bar{H}_{2}}^{\bar{H}_{1}} \bar{U}(\bar{X}, \bar{Y}, \bar{t}) d \bar{Y}
$$

where $\bar{H}_{1}$ and $\bar{H}_{2}$ are functions of $X, t$.

The rate of volume flow in the wave frame is given by

$$
q=\int_{\bar{H}_{2}}^{\bar{H}_{1}} \bar{u}(\bar{x}, \bar{y}) d \bar{y},
$$

where $h_{1}$ and $h_{2}$ are the functions of $x$ alone. If we substitute equation (1.7) into (2.1) and make use of (2.2), it is found that the related rates of volume flow is:

$$
Q=q+c_{1} \bar{H}_{1}-c_{1} \bar{H}_{2}
$$

The time-mean flow over a period $T$ at a fixed position $X$ is defined as:

$$
Q^{*}=\frac{1}{T} \int_{0}^{T} Q d t
$$

Substituting (2.3) into (2.4), and integrating, we get:

$$
Q^{*}=q+c_{1} d_{1}+c_{1} d_{2}
$$

On defining the dimensionless time-mean flows $\bar{Q}$ and $\bar{q}$ in the fixed and wavy frame respectively as:

One finds that (2.5) may be written as:

$$
\bar{Q}=\frac{Q^{*}}{c_{1} d_{1}} \quad \text { and } \quad \bar{q}=\frac{q}{c_{1} d_{1}},
$$

$$
\bar{Q}=\bar{q}+1+d .
$$

From Eq. (2.23), with the help of Eqs. (3.1)-(3.7), we get the pressure gradient. The pressure rise $\Delta p$ and the friction force $F_{\lambda}$ (at the walls) for a channel of length $L$, in their non-dimensional forms, are given by

$$
\begin{aligned}
\Delta p & =\int_{0}^{1} \frac{\partial p}{\partial x} d x, \\
F_{\lambda}^{h_{1}} & =\int_{0}^{1}\left(-h_{1}^{2} \frac{\partial p}{\partial x}\right) d x . \\
F_{\lambda}^{h_{2}} & =\int_{0}^{1}\left(-h_{2}^{2} \frac{\partial p}{\partial x}\right) d x .
\end{aligned}
$$

The integrals in equations (3.9)-(3.10) are not integrable in closed form, they are evaluated numerically using a ready package of Nintegrate Library from Mathematica 7. 
Nintegrate uses an algorithm called 'integration strategies' that attempts to compute an integral which satisfy user specified precision or accuracy goals.

The corresponding stream functions $\left(u=\frac{\partial \psi}{\partial y}, v=-\frac{\partial \psi}{\partial x}\right)$ are evaluated with boundary condition $\psi=0$ at $z=\frac{1}{2}\left(h_{1}+h_{2}\right)$

\section{Graphical results and discussion}

Here we discuss the quantitative effects of sundry parameters used in analysis with the help of graphs and results. First, let us discuss the variations observed for the pressure rise distributions versus the time average flux $(\bar{Q})$, for different values of the local nanoparticle Grashof number $\left(B_{r}\right)$, local thermal Grashof number $\left(G_{r}\right)$, porosity parameter $(K)$, thermophoresis parameter $\left(N_{t}\right)$, non-uniform parameters $\left(k_{1}\right)$ and $\left(k_{2}\right)$, Hall parameter $(M)$. It is noticed in Figure 2(a) that with an increase in the local nanoparticle Grashof Number $\left(B_{r}\right)$, pressure rise reduces but Figure 2(b) shows the opposite behavior and pressure rise increases with the increase in the thermal Grashof number $\left(G_{r}\right)$. At the same time Figure 2(c) shows the pressure rise plunges with the increase in porosity parameter $(K)$, showing anticlockwise rotation in the graph. Figure 2(d) shows that with an increase in the thermophoresis number $\left(N_{t}\right)$ pressure rise reduces. Figures 2(e) and 2(f) give the effect of the non-uniform parameters $\left(k_{1}\right)$ and $\left(k_{2}\right)$ on the pressure rise in the flow, and show that it plunges with the increase of the non-uniform parameters $\left(k_{1}\right)$ and $\left(k_{2}\right)$ showing anticlockwise rotation in the graph. In the retrograde pumping region the pressure rise decreases with an increase in non-uniform parameters $\left(k_{1}\right)$ and $\left(k_{2}\right)$. Finally, in Figure 2(g) the Hall parameter $(M)$ gives the opposite effect to the porosity parameter $(K)$, where the pressure rise increases in the retrograde quadrant with an increase in Hall parameter $(M)$ and goes in clockwise rotation.
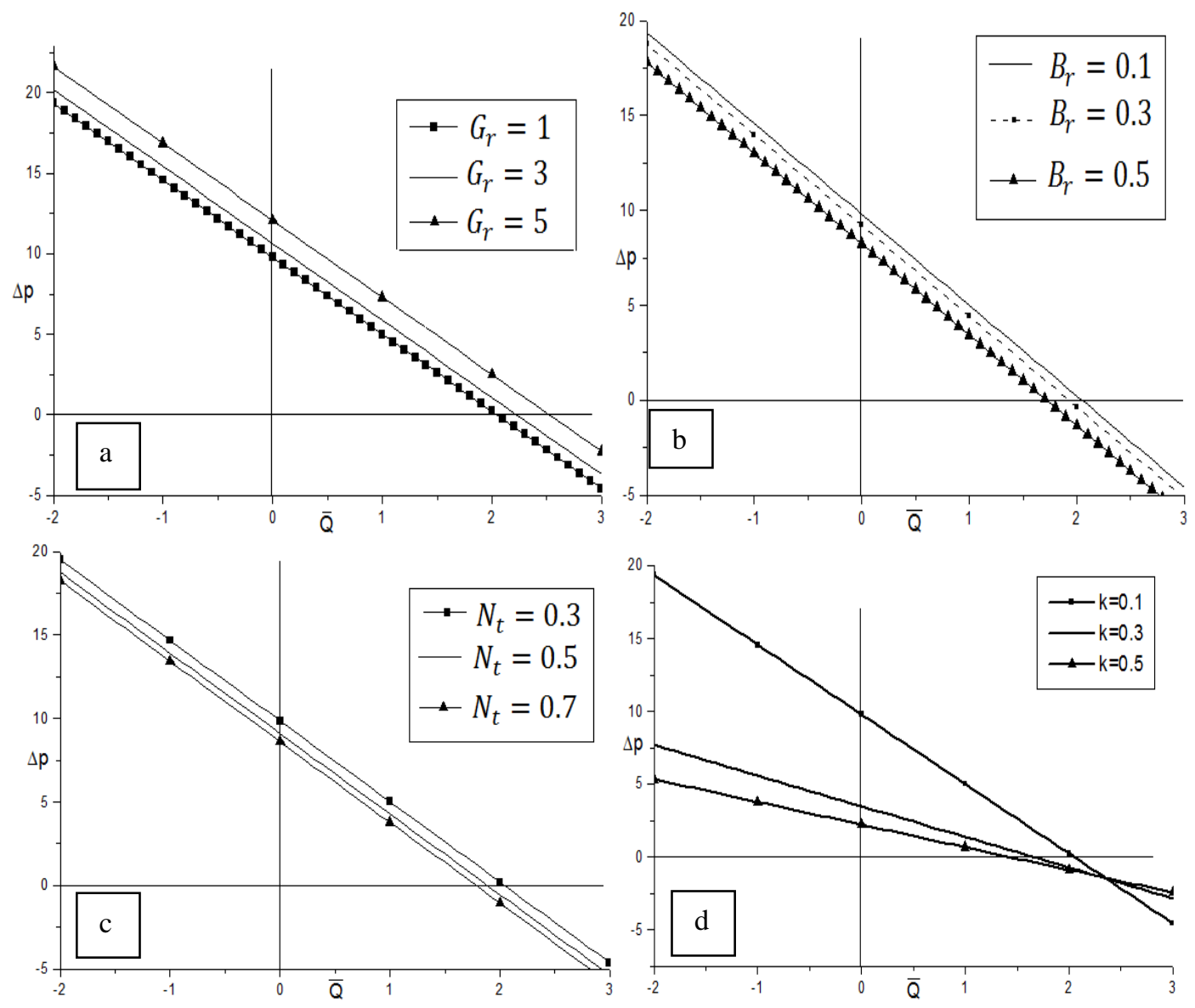

Figure 2. Variation of pressure rise over the length versus $\bar{Q}$. 

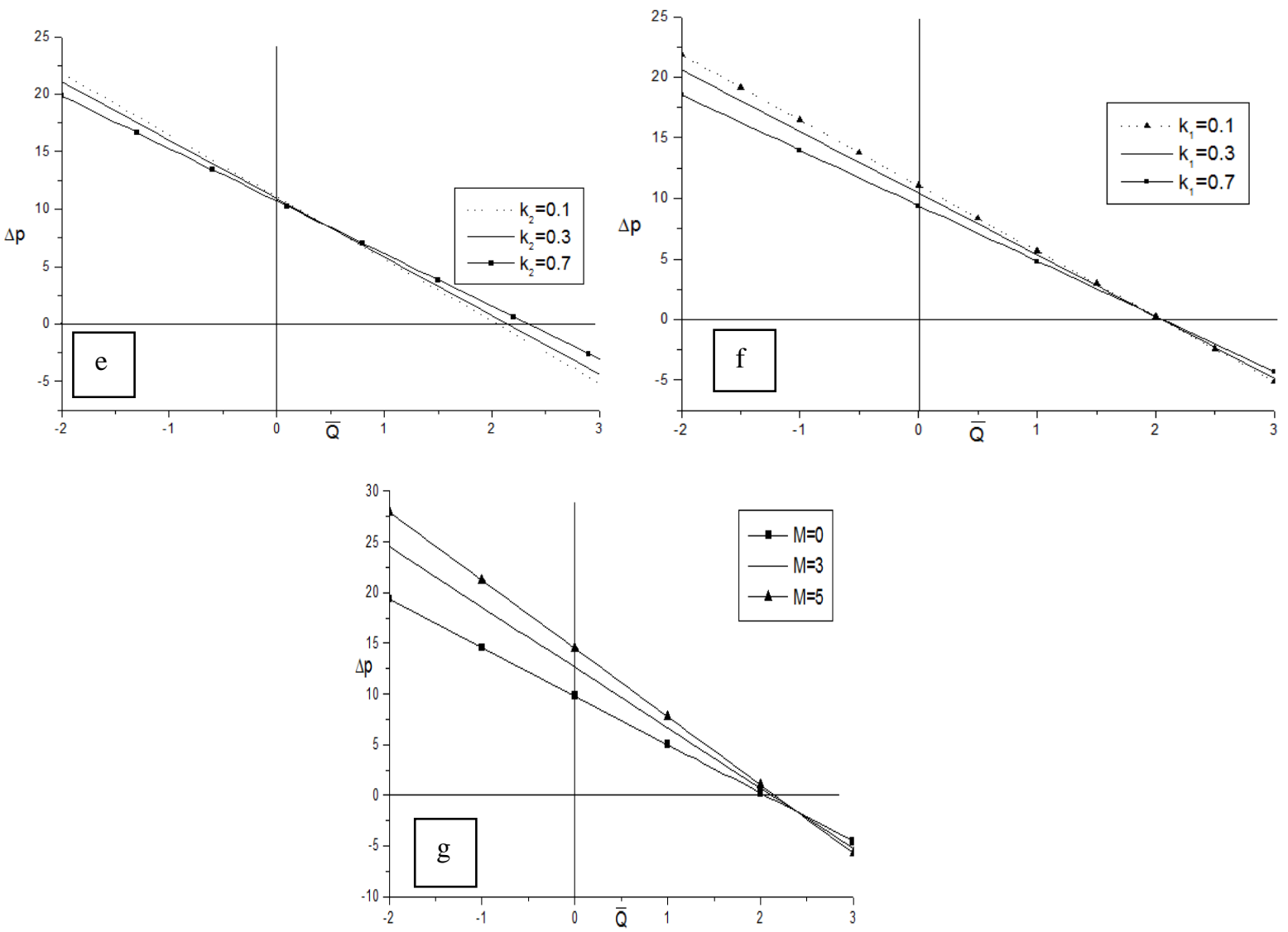

Figure 2. Contd.

A fluid particle that moves with a velocity that is different from its neighbours will be subjected to a frictional force, such forces also exist between a particle and a rigid boundary. The friction forces $F_{\lambda}^{1}$ and $F_{\lambda}^{2}$ on the right and left walls respectively can be obtained by integral evaluations of the analytic results gained for the pressure gradient $\frac{d p}{d x}$. The average of the right friction force (on the right flexible wall) as well as the average of the left friction force (on the left flexible wall) are plotted in Figure 3
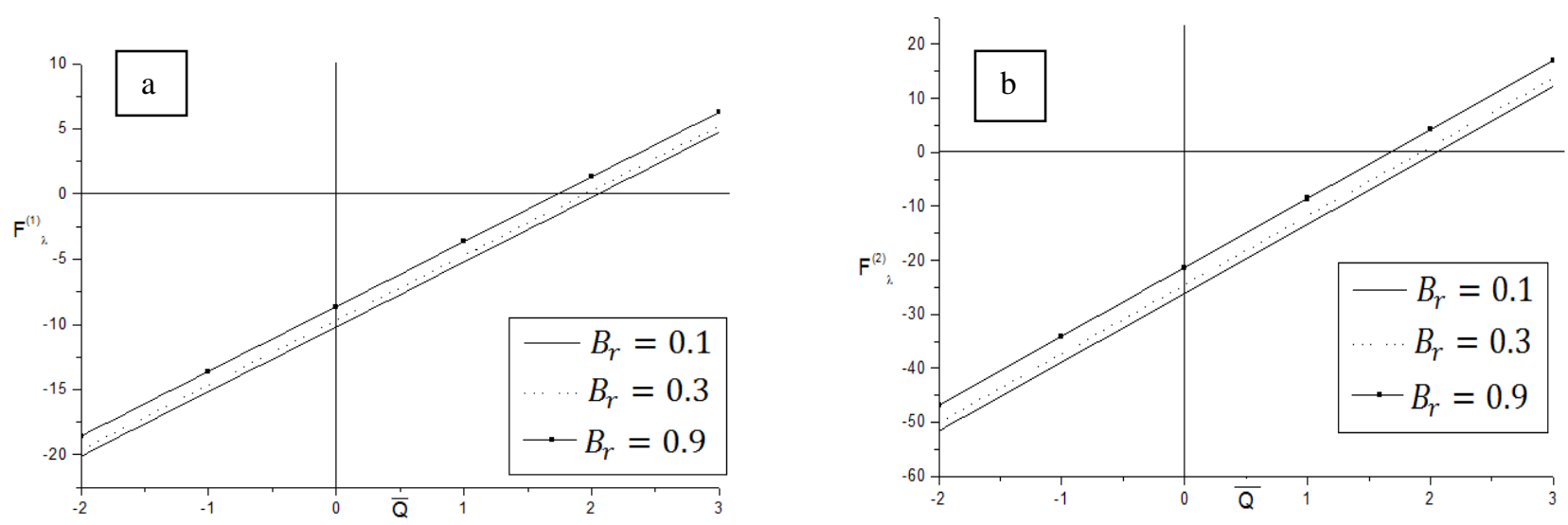

Figure 3. Variation of frictional forces. 

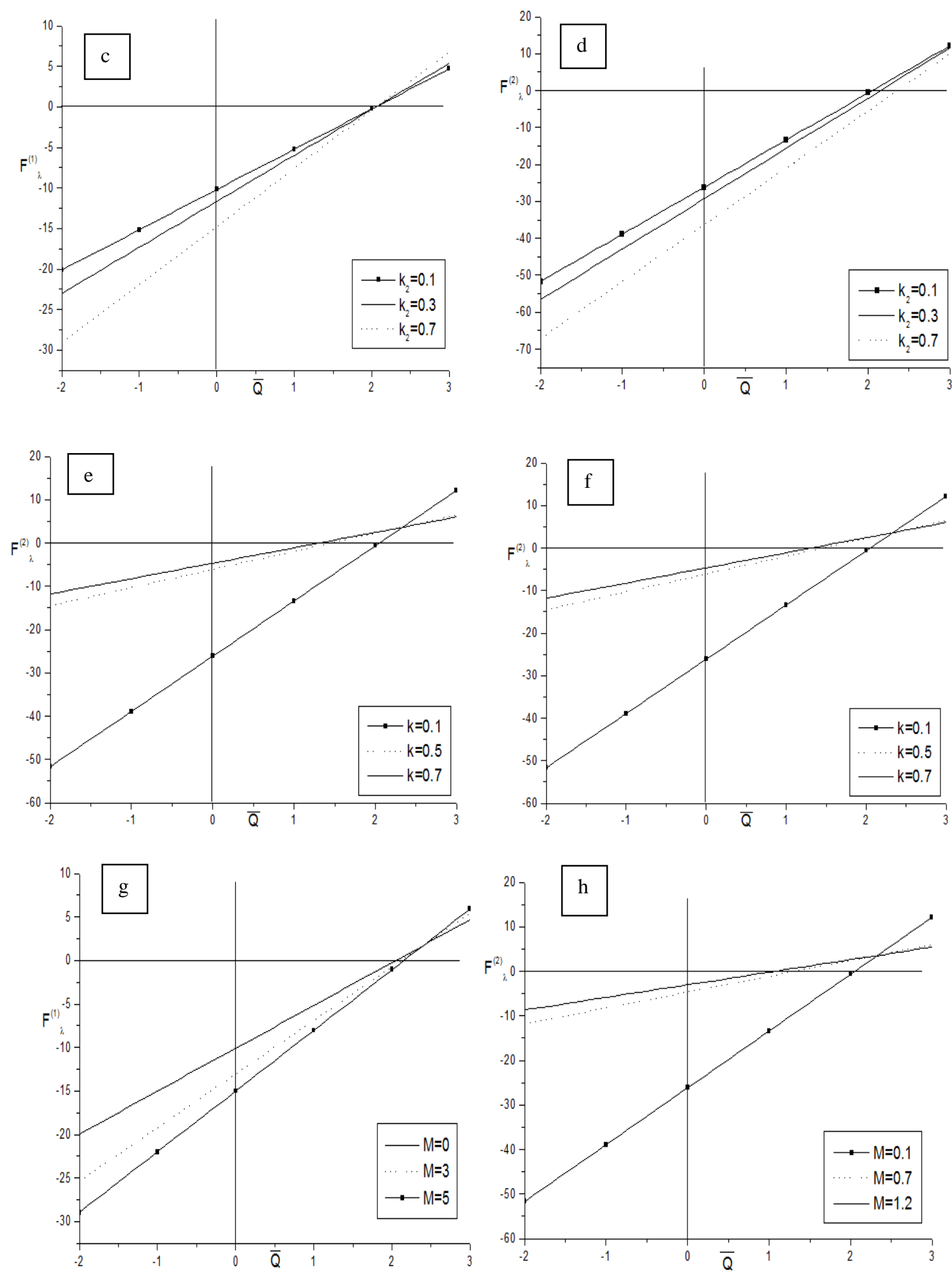

Figure 3. Contd.

We notice from these figures that the right and left wall friction forces have the opposite behavior as compared to the pressure rise. The right friction force behaves similarly to the left friction force for the same values of the parameters; however, the absolute value of the right friction force is greater than that of the left friction force at the same values of the parameters.

Trapping is the development and transport of an internally circulating bolus of fluid. In this bolus a fluid travels along the wave as it propagates. The effect of the Hall parameter $(M)$ on the trapping can be observed through Figure 4 where the size of the bolus reduces with an increase in Hall parameter $(M)$. The influences of phase 


\section{COMBINED EFFECTS OF HALL CURRENT AND HEAT TRANSFER}

difference $(\phi)$, porosity parameter $(K)$, wall temperature ratio $m_{0}$, non-uniform parameters $\left(k_{1}\right)$ and $\left(k_{2}\right)$, Hall parameter $(M)$ and thermophoresis parameter $\left(N_{t}\right)$ are analyzed through Figures (4) and (5). We notice that the size of the trapped bolus increases with an increase in porosity parameter $(K)$ and wall temperature ratio $m_{0}$ but it reduces with an increase in thermophoresis parameter $\left(N_{t}\right)$ and the Hall parameter $(M)$. We can also observe that streamlines get shifted due to the change in the values of phase difference $(\phi)$ and wall temperature ratio $m_{0}$, and non-uniform parameter $\left(k_{1}\right)$ and $\left(k_{2}\right)$.
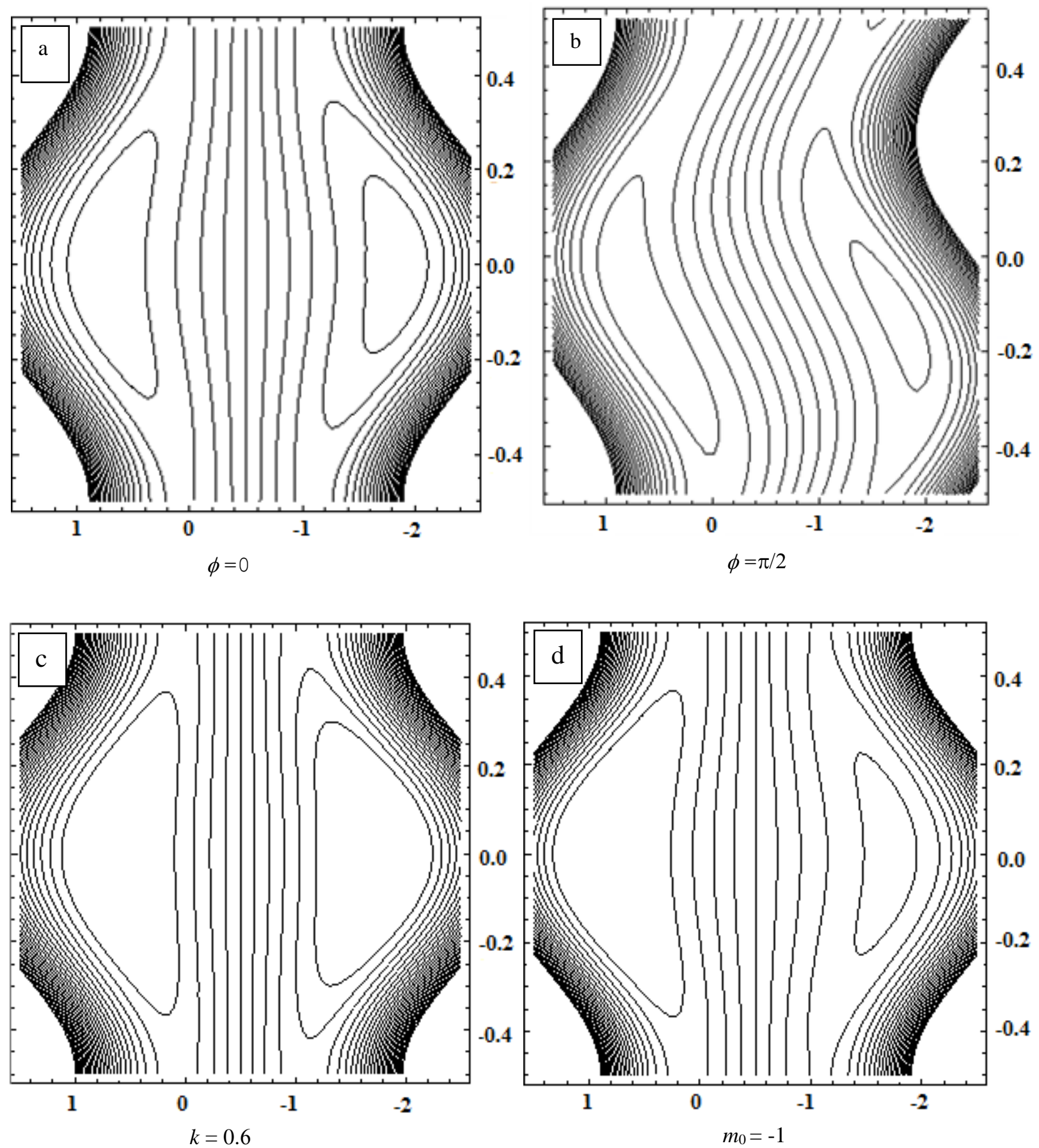

Figure 4. Streamlines for different values of $\phi, k, m_{0}$ when $N_{t}=0.2, N_{b}=0.4, Q=0.4, B_{r}=0.4, G_{r}=0.6$, $m=0, k_{1}=0, k_{2}=0$. 
Figure 6 depicts the variations of temperature profile $(\theta)$ and nanoparticle volume fraction $(\sigma)$ for different values of the thermophoresis parameter $\left(N_{t}\right)$, wall temperature ratio $m_{0}$ and non-uniform parameters $\left(k_{1}\right)$ and $\left(k_{2}\right)$. It is seen in Figures $6(\mathrm{c}), 6(\mathrm{e})$ and $6(\mathrm{~g})$ that the heat distribution $(\theta)$ increases with an increase in non-uniform parameter $\left(k_{2}\right)$, thermophoresis parameter $\left(N_{t}\right)$ and temperature ratio $m_{0}$. On the other hand, Figures $6(\mathrm{~b}), 6(\mathrm{~d}), 6(\mathrm{f})$, and 6(h) show two different behaviour of the nanoparticles volume fraction $(\sigma)$ profiles. It decreases with the increase of the thermophoresis parameter when $N_{t}<0$, wall temperature ratio $m_{0}$ and non-uniform parameters $\left(k_{1}\right)$ and $\left(k_{2}\right)$. An opposite trend is observed when $N_{t}>0$.
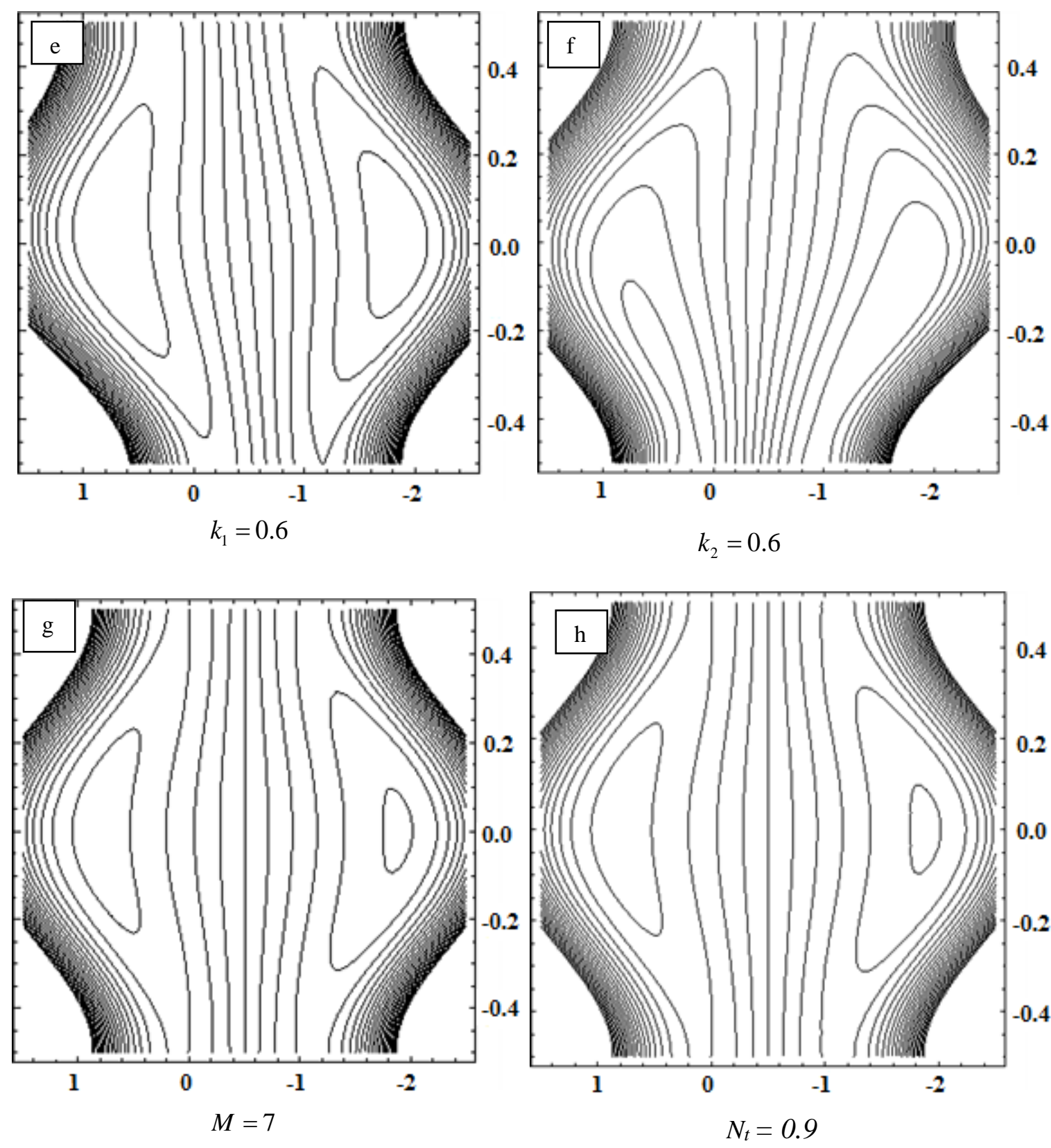

Figure 5. Streamlines for the different values of $k_{1}, k_{2}, M, N_{t}$ when $N_{b}=0.4, Q=0.4, B_{r}=0.4, G_{r}=0.6$, $k=0.2, m_{0}=2$. 

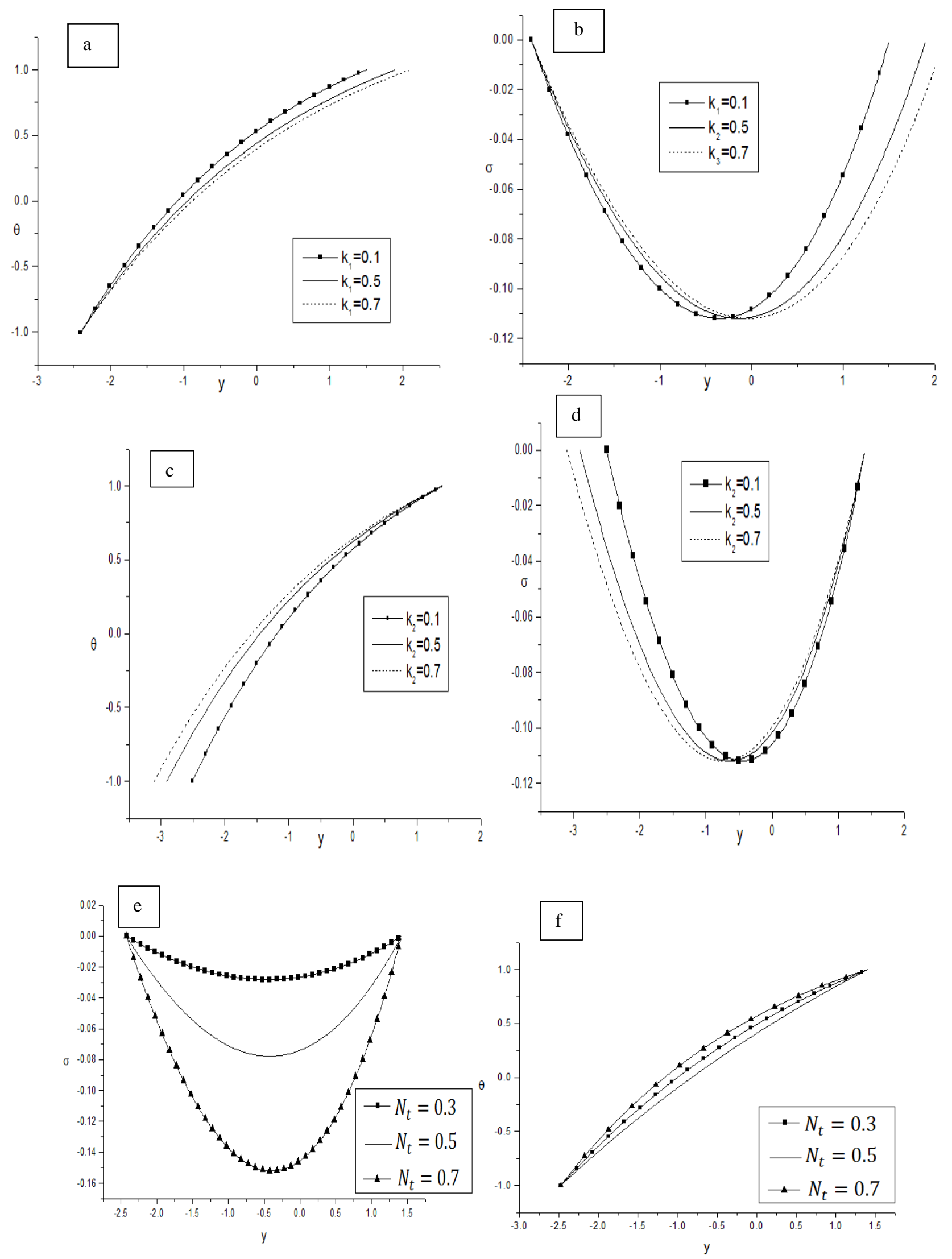

Figure 6. Concentration profile $\sigma$ and heat distribution $\theta$. 

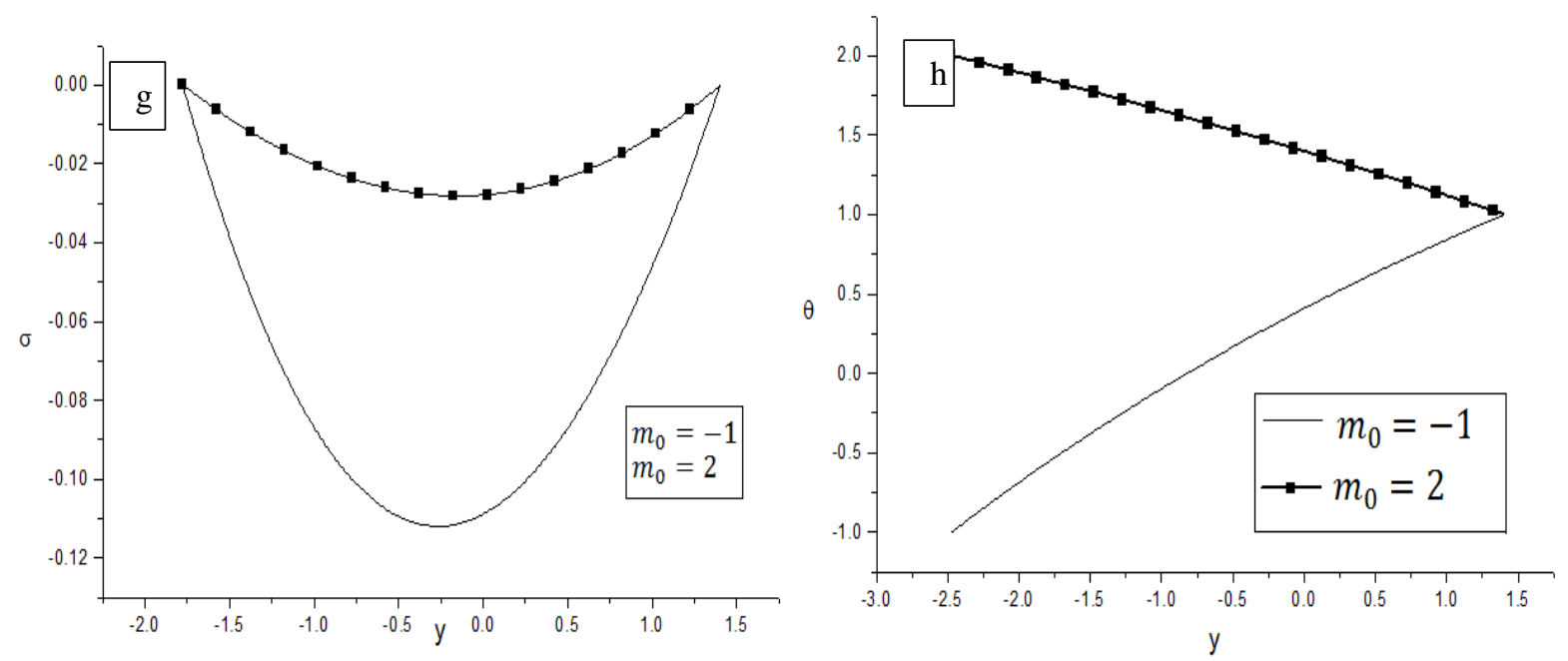

Figure 6. Contd.

\section{Conclusion}

The pressure rise reduces with an increase in the Hartmann number $(M)$ and Thermophoresis parameter $\left(N_{t}\right)$. It increases with the increase of the local nanoparticle Grashof number $\left(B_{r}\right)$, local temperature Grashof number $\left(G_{r}\right)$ and $N_{b}$. All these behaviors are in agreement with those reported by Kothandapani and Prakash [25]. The temperature distribution profile $\theta$ increases whereas nanoparticle concentration decreases with the increase of $N_{t}$ and $N_{b}$.

\section{References}

1. Mc Whirter, J.D., Crawford, M.E. and Klein, D.E. Magnetohydrodynamic flows in porous media II: experimental results. Fusion Techno., 1998, 34, 187-197.

2. Geindreau, C. and Auriault, J.L. Magnetohydrodynamic flows in porous media. J. Fluid Mech., 2002, 466, 343363.

3. Latham, T.W. Motion in a Peristaltic Pump, M.S. Thesis, MIT-Press, Cambridge, Mass, USA, 1966.

4. Fung, Y.C. and Yih, C.S. Peristaltic transport. J. App. Mech., 1968, 35, 669-675.

5. Shapiro, H., Jaffrin, M.Y. and Weinberg, S.L. Peristaltic pumping with long wavelengths at low Reynolds number. J. Fluid Mech., 1969, 37, 799-825.

6. Jaffrin, M.Y. and Shapiro, A.H. Peristaltic pumping. Annual Rev. Fluid Mech., 1971, 3, 13-36.

7. Srivastava, L.M. and Srivastava, V.P. Peristaltic transport of blood: Casson model II. J. Biomech., 1984, 17, 821829.

8. El Shehawey, E.F., Eldabe, N.T., Elghazy, E.M. and Ebaid, A. Peristaltic transport in an asymmetric channel through a porous medium. App. Math. Comp. 2006, 182, 140-150.

9. Vajravelu, K., Radhakrishnamacharya, G. and Radhakrishnamurty, V. Peristaltic flow and heat transfer in a vertical porous annulus, with long wave approximation. Int. J. Non-Linear Mech., 2007, 42, 754-759.

10. Srinivas, S. and Kothandapani, M. The influence of heat and mass transfer on MHD peristaltic flow through a porous space with compliant walls. App. Math. Comp., 2009, 213, 197-208.

11. Srinivas, S. and Gayathri, R. Peristaltic transport of a Newtonian fluid in a vertical asymmetric channel with heat transfer and porous medium. App. Math. Comp., 2009, 215, 85-196.

12. Attia, H.A. Unsteady hartmann flow with heat transfer of a viscoelastic fluid considering the hall effect. Canadian J. Phys., 2004, 82, 127-139.

13. Asghar, S., Mohyuddin, M.R. and Hayat, T. Effects of Hall current and heat transfer on flow due to a pull of eccentric rotating disks. Int. J. Heat Mass Trans., 2005, 48, 599-607.

14. Hayat, T., Ali, N. and Asghar, S. Hall effects on peristaltic flow of a Maxwell fluid in a porous medium. Phys. Lett. A., 2007, 363, 397-403.

15. Abo-Eldahab, E.M., Barakat, E.I. and Nowar, K.I. Hall currents and ion-slip effects on the MHD peristaltic transport. Int. J. App. Math. Phys., 2010, 2, 113-123.

16. Abo-Eldahab, E.M., Barakat, E.I. and Nowar, K.I. Effects of Hall and ion-slip currents on peristaltic transport of a couple stress fluid. Int. J. App. Math. Phys., 2010, 2, 145-157.

17. Akbar, N.S. and Nadeem, S. Influence of heat transfer on peristaltic transport of a Johnson-Segalman fluid in an inclined asymmetric channel. Commun. Nonlin. Sci. Numer. Simulat., 2010, 15, 2860-2877. 
18. Ali, N., Sajid, M., Javed, T. and Abbas, Z. Heat transfer analysis of peristaltic flow in a curved channel. Int. J. Heat Mass Trans., 2010, 53, 3319-3325.

19. Mekheimer, K.S., Saleem, N., Hayat, T. and Hendi, A.A. Simultaneous effects of induced magnetic field and heat and 16 mass transfer on the peristaltic motion of second-order fluid in a channel. Int. J. Numer. Meth. Fluid Flow., 2012, 70, 342-358.

20. Mekheimer, K.S. and Abdel-Wahab, A.N. Net annulus flow of a compressible viscous liquid with peristalsis. $J$. Aerospace Eng., 2012, 25, 660-669.

21. Abd El-Maboud, Y. and Mekheimer, K.S. Non-linear peristaltic transport of a second-order fluid through a porous medium. App. Math. Model., 2010, 35, 2695-2710.

22. Mekheimer, K.S., Husseny, S.A. and Abd El-Maboud, Y. Effects of heat transfer and space porosity on peristaltic flow in a vertical asymmetric channel. Numer. Meth. Partial Diff. Equa. Journal, 2010, 26, 747-770.

23. Gharsseldien, Z.M., Mekheimer, K.S. and Awad, A.S. The influence of slippage on trapping and reflux limits with peristalsis through an asymmetric channel. App. Bionics Biomec., 2010, 7, 95-108.

24. Srinivas, S. and Muthuraj, R. Effects of chemical reaction and space porosity on MHD mixed convective flow in a vertical asymmetric channel with peristalsis. Math. Comp. Model., 2011, 54, 1213-1227.

25. Kothandapani, M. and Prakash, J. Effect of radiation and magnetic field on peristaltic transport of nanofluid through a porous space in a tapered asymmetric channel. J. Magnet. Magnet. Mater. 2015, 378, $152-163$.

\section{Appendix}

$$
\begin{aligned}
& A_{1}=\frac{1-m_{0}}{\left(e^{-s_{1} N_{b} h_{1}}-e^{-s_{1} N_{b} h_{2}}\right)}, \quad A_{2}=\frac{1}{k}+\frac{M}{1+m_{1}^{2}}, \\
& s_{1}=\frac{N_{t}\left(1-m_{0}\right)}{N_{b}\left(h_{1}-h_{2}\right)} \\
& c_{2}=c_{7}+\frac{e^{-s_{1} N_{b} h_{1}}}{A_{2}}\left(-G_{r} A_{1}+\frac{B_{r} N_{t} A_{1}}{N_{b}}\right), c_{3}=\frac{B_{r} \mathrm{~s}_{1}}{A_{2}} \\
& c_{4}=-c_{8}\left(-1-\left(\frac{e^{-s_{1} h_{1} N_{b}}}{A_{2}}+\frac{e^{-s_{1} h_{1} N_{b}}}{-A_{2}+s_{1}^{2} N_{b}^{2}}\right)\left(-A_{1} G_{r}+\frac{A_{1} B_{r} N_{t}}{N_{b}}\right)-\frac{G_{r}-p}{A_{2}}\right)- \\
& c_{9}\left(-1-\left(\frac{e^{-s_{1} h_{1} N_{b}}}{A_{2}}+\frac{e^{-s_{1} h_{2} N_{b}}}{-A_{2}+s_{1}^{2} N_{b}^{2}}\right)\left(-A_{1} G_{r}+\frac{A_{1} B_{r} N_{t}}{N_{b}}\right)-\frac{G_{r}-B_{r} s_{1} h_{1}+B_{r} s_{1} h_{2}-p}{A_{2}}\right), \\
& c_{5}=e^{-\sqrt{A_{2} h_{1}}}\left(-1-\left(\frac{e^{-s_{1} h_{1} N_{b}}}{A_{2}}+\frac{e^{-s_{1} h_{1} N_{b}}}{-A_{2}+s_{1}^{2} N_{b}^{2}}\right)\left(-A_{1} G_{r}+\frac{A_{1} B_{r} N_{t}}{N_{b}}\right)-\frac{G_{r}-p}{A_{2}}\right)+ \\
& c_{7}\left(e^{\sqrt{A_{2}} h_{2}}\left(-1-\left(\frac{e^{-s_{1} h_{1} N_{b}}}{A_{2}}+\frac{e^{-s_{1} h_{1} N_{b}}}{-A_{2}+s_{7}^{2} N_{b}^{2}}\right)\left(-A_{1} G_{r}+\frac{A_{1} B_{r} N_{t}}{N_{b}}\right)-\frac{G_{r}-p}{A_{2}}\right)-\right. \\
& \left.e^{\sqrt{A_{2}} h_{1}}\left(-1-\left(\frac{e^{-s_{1} h_{1} N_{b}}}{A_{2}}+\frac{e^{-s_{1} h_{2} N_{b}}}{-A_{2}+s_{1}^{2} N_{b}^{2}}\right)\left(-A_{1} G_{r}+\frac{A_{1} B_{r} N_{t}}{N_{b}}\right)-\frac{G_{r}-B_{r} s_{1} h_{1}+B_{r} s_{1} h_{2}-p}{A_{2}}\right)\right), \\
& c_{6}=\frac{1}{N_{b}^{2} s_{1}^{2}-A_{2}}\left(-G_{r} A_{1}+\frac{B_{r} N_{t} A_{1}}{N_{b}}\right) \text {, } \\
& c_{7}=\frac{-p+G_{r}-B_{r} s_{1} h_{1}}{A_{2}} \\
& c_{8}=e^{\left(h_{2}+2 h_{1}\right) \sqrt{A_{2}}} c_{7}, \quad c_{9}=e^{3 h_{1} \sqrt{A_{2}}} c_{7}
\end{aligned}
$$

